\title{
Motor attempt EEG paradigm as a diagnostic tool for disorders of consciousness
}

\author{
Christoph Schneider ${ }^{1}$, Serafeim Perdikis ${ }^{1}$, Member, IEEE, Marina Silva ${ }^{2}$, Jane Jöhr ${ }^{2,3}$, \\ Alexander Pincherle ${ }^{2}$, José del R. Millán ${ }^{1}$, Fellow, IEEE and Karin Diserens ${ }^{2}$
}

\begin{abstract}
To investigate whether a motor attempt EEG paradigm coupled with functional electrical stimulation can detect command following and, therefore, signs of conscious awareness in patients with disorders of consciousness, we recorded nine patients admitted to acute rehabilitation after a brain lesion. We extracted peak classification accuracy and peak session discriminant power (PSDP) and we assessed their correlation to the established coma recovery scale revised (CRS-R) and the agreement with diagnosis based on the novel motor behavior tool (MBT). Only PSDP correlated significantly with CRS-R and it also outperformed peak accuracy regarding the MBT. We conclude that PSDP might be more suitable than accuracy to complement CRS-R and MBT in evaluating ambiguous cases and in detecting cognitive motor dissociation.
\end{abstract}

\section{INTRODUCTION}

After severe brain lesions patients are likely to experience disorders of consciousness (DOC), which are defined as conditions of compromised environmental and self-awareness. Depending on the degree of awareness (often after emerging from coma), these patients are classified into unresponsive wakefulness syndrome (UWS), also referred to as vegetative state (VS), and minimally conscious state (MCS) [1]. Early, accurate diagnosis and prognosis of outcome are critical for the medical treatment the patients receive in acute rehabilitation and for informed end-of-life decisions that may have to be made. Assessment of awareness is conventionally done through behavioral tests [2], most notably the Glasgow Coma Scale (GCS). Despite fruitful efforts to define improved clinical scales [3], among which the nowadays well established Coma Recovery Scale-Revised (CRS-R) [4], such behavioral assessment tools still suffer well-documented limitations, in particular regarding their inability to differentiate cases in the lower spectrum of awareness and their dependence on the existence of residual motor functionality [5].

To alleviate these limitations, neuroimaging-based approaches have been intensively studied in recent years as complementary or standalone diagnostic and prognostic tools for DOC [1], [6]. Importantly, brain imaging has allowed the identification of what is now widely accepted as a distinct

\footnotetext{
${ }^{1}$ Defitech Chair in Brain-Machine Interface (CNBI), École Polytechnique Fédérale de Lausanne (EPFL), Switzerland; Corresponding author: José del R. Millán jose.millan@epfl.ch

${ }^{2}$ Acute Neurorehabilitation Unit, Division of Neurology, Department of Clinical Neurosciences, Centre Hospitalier Universitaire Vaudois (CHUV), Switzerland

${ }^{3}$ Division of Neurorehabilitation and Neuropsychology, Department of Clinical Neurosciences, Centre Hospitalier Universitaire Vaudois (CHUV), Switzerland
}

case, namely, cognitive motor dissociation (CMD). CMD encompasses patients that, due to a complete absence of meaningful motor output, would be classified as UWS or marginal MCS based on clinical criteria, but nevertheless exhibit command-following behavior evident in brain signal responses [7]. Although functional brain imaging like Positron Emission Tomography (PET) [8] and functional Magnetic Resonance Imaging (fMRI) have provided the first breakthroughs [9] and are still preferred for their high spatial resolution, electroencephalography (EEG)-based assessment of consciousness offers a number of practical advantages (portability, low price, less contraindications), thus holding great promise for bedside detection of awareness [10]-[12]

Both, task-free [13] and task-dependent [10], [11] paradigms have yielded promising results. But while the former might be applicable also in the presence of reduced cognitive ability and compromised sensory pathways, taskdependent paradigms relying either on evoked potentials [11] or motor tasks [10] are promising for eventually establishing brain-computer interface (BCI) communication with CMD patients in complete locked-in syndrome (CLIS) [14].

Here, we provide a preliminary evaluation of the diagnostic potential of a motor attempt EEG paradigm [1], [10], [14] coupled with functional electrical stimulation (FES) to provide contingent, rich, afferent feedback and increase patient vigilance. Preliminary results with acute DOC patients suggest that a discriminancy index quantifying the patients' ability to modulate sensorimotor rhythms (SMRs) might be a more suitable predictor of consciousness compared to the widely used classification accuracy. We further discuss these findings with respect to independent clinical observations made by means of the MBT, a novel clinical instrument designed to detect signs of covert consciousness, reveal CMD patients and refine the prognosis of outcome [15].

\section{MATERIAls AND METHODS}

\section{A. Patients and data}

We assessed 9 patients (S1-S9, 2 female, age $53 \pm 19$ ) admitted to the Acute Neurorehabilitation Unit (NRA) of the Lausanne University Hospital (CHUV), Switzerland, four after hemorrhagic or ischemic stroke and five after traumatic brain lesion. Written consent to participate in the study was acquired from the relatives. The experimental protocol (№ 142/09) was approved by the ethical commission of the canton of Vaud, Switzerland and adheres to the principles of the declaration of Helsinki. All patients underwent repeated behavioral CRS-R scoring during their hospital stay by 
medical doctors. Since the CRS-R score was not always available on the exact day of the EEG session(s), the reported scores were linearly interpolated using the nearest two data points, as needed. The longest time between clinical scoring and the nearest EEG session was 4 days. MBT evaluation (complementing CRS-R measurements) was done before patient admission to the NRA and identified patient S3 as UWS and all remaining patients as CMD. Data of another 3 enrolled patients had to be discarded due to insufficient quality. In total, we report on 45 runs in 14 recording sessions of 9 different patients.

\section{B. Experimental setup}

During the EEG session, patients were lying in their bed, except for the second session of S4 where the patient was seated on a chair as required by the caregivers. EEG signals were recorded with a 16-channel active-electrode montage in standard 10-10 positions covering the motor cortex (see Fig. 1C). The amplifiers used were a g.USBamp sampling at $512 \mathrm{~Hz}$ and a g.Nautilus wireless amplifier sampling at $500 \mathrm{~Hz}$ (g.tec, Schiedlberg, Austria). Biphasic neuromuscular electrical stimulation was delivered with one bipolar channel through a Motionstim 8 device (MEDEL, Hamburg, Germany). The FES train was delivered at $35 \mathrm{~Hz}$, lasted $2 \mathrm{~s}$ and consisted of a $1 \mathrm{~s} \mathrm{ramp}$ with linearly increasing pulse width from 10 to $500 \mu \mathrm{s}$, followed by $1 \mathrm{~s}$ of continuous stimulation.

\section{Experimental protocol}

Each patient participated in 1 to 3 EEG sessions (average 1.5), which comprised 2 to 4 runs (average 3.1). Each run was around 6 minutes long and consisted of 15 motor attempt trials randomly interleaved with 15 rest trials. Before each session, patients were woken up and given verbal instructions to either attempt unilateral hand movement or rest following the corresponding auditory cues. Two FES electrodes were placed on the extensor digitorum communis of the same arm forming a single bipolar channel and the FES amplitude was adjusted so as to achieve a full hand extension movement. FES amplitudes varied between 8 and $15 \mathrm{~mA}$. Each trial started with an auditory cue played via inear headphones, which prompted (in French) the patient to either move ("Bougez") or to not move ("Ne bougez pas"). Each trial lasted 5 seconds, starting with the auditory cue. Motor attempt trials were followed by FES. The inter-trial period had variable length between 3 and 4 seconds. The experimenter was shown the protocol and the EEG-signals on a computer screen.

\section{Data analysis}

The recorded EEG signals were spatially filtered with a small Laplacian derivation. Noisy channels were excluded from further analysis. Then the signals were spectrally filtered with a 5th order Butterworth bandpass filter with cutoff frequencies at 1 and $48 \mathrm{~Hz}$ to eliminate slow signal drifts and the power line interference. Trials with artifacts in the EEG signal were automatically detected and removed from further analysis if their absolute means, maxima or mean derivatives

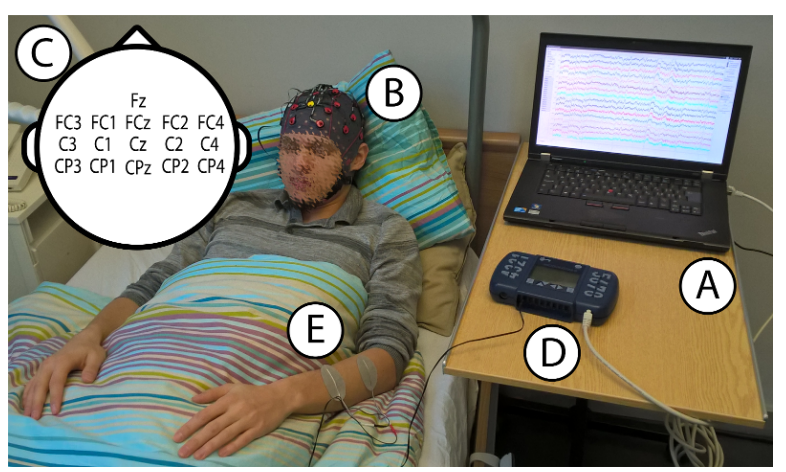

Fig. 1. Bedside experimental setup: (A) Stimulus presentation and recording computer, (B) EEG cap with integrated wireless amplifier, (C) EEG channel layout, (D) computer controlled FES device, (E) FES electrodes.

exceeded three median absolute deviations (MAD). Channel $\mathrm{Fz}$ was excluded form all analysis as it is susceptible to ocular and facial movements.

For each trial, we extracted the time span from one second after the cue (to avoid potential interference from signals evoked by the auditory cue) to the end of the trial. The power spectral density (PSD) of the EEG signal was computed for each channel individually in $1 \mathrm{~s}$ long sliding windows shifted by $62.5 \mathrm{~ms}$. The extracted frequency bins for the features were $2 \mathrm{~Hz}$ wide and centered at even numbers between 8 and $30 \mathrm{~Hz}$, covering both the $\mu$ and $\beta$ EEG bands.

For each PSD feature we calculated the coefficient of determination $r^{2}$ between the feature values and their corresponding class labels within each run. This distance metric allows a quantitative assessment of which channel-frequency pairs exhibit good discriminant power between the SMR EEG activity elicited by the two tasks, motor attempt and rest. Subsequently, we computed single-sample classification accuracies using 10-fold cross validation and a linear discriminant analysis classifier. The partitioning in folds was done at the trial level to avoid accuracy overestimation resulting from the high autocorrelation of EEG within the same trial. Inside each partition, the five best features were selected based on their $r^{2}$ discriminancy, excluding features with a correlation of more than 0.9 between them.

Further, we defined a new measure peak session discriminant power (PSDP) as the maximum $r^{2}$-discriminancy across all features in each session.

\section{RESUlts}

As Fig. 2A shows, PSDP is significantly correlated to CRS-R $(r=0.638, p=0.0198)$ and the correlation was robust in a leave-one-out test $(r=0.613 \pm 0.045, p=$ $0.028 \pm 0.012$ ). Additionally, in spite of a quite uniform distribution of CRS-R values, PSDP values seem to group in a high $\left(r^{2}=0.074 \pm 0.004\right)$ and a low cluster $\left(r^{2}=\right.$ $0.033 \pm 0.004)$. On the contrary, peak accuracy does not correlate with CRS-R $(r=0.310, p=0.280$, Fig. 2B).

Fig. 3 shows the peak (across all runs) single-sample classification accuracy per session between the classes motor attempt and rest. The mean classification accuracy was 

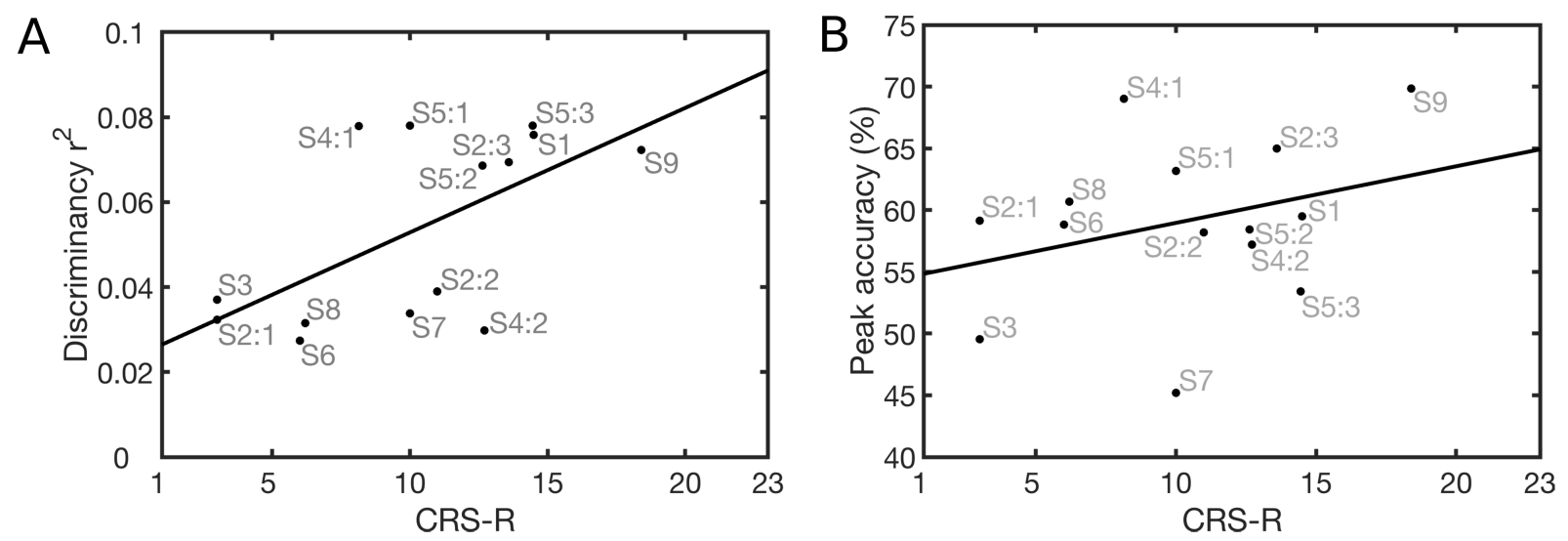

Fig. 2. (A) PSDP and (B) peak accuracy against CRS-R for all patients and sessions. Linear fits are illustrated with solid black lines. For patients with multiple sessions, the session is denoted as number after the colon.

$51.77 \% \pm 7.90 \%$. Only one (S4:1) out of 14 recording sessions contained a run that surpassed the $99 \%$ chance level, obtained individually for each run by 1000 random permutation computations. No session contained two runs above chance level.

Motor execution and motor imagery leads to a decrease in spectral power around the Rolandic fissure, notably in the $\mu$ $(8-12 \mathrm{~Hz})$ and $\beta$ rhythm $(13-30 \mathrm{~Hz})$ [16]. We observed eventrelated desynchronization (ERD) in both spectral bands for our experiment (see Fig. 4). The ERD amplitude for sessions with high PSDP is on average four times higher than for sessions with low PSDP, a finding consistent with higher $r^{2}$ discriminancy in this group.

\section{Discussion}

Task-dependent, EEG-based studies for awareness evaluation conventionally impose a requirement of above chance level classification peak accuracy to diagnose a patient as aware [12]. However, MCS and CMD patients typically exhibit weak and intermittent SMR modulation which can easily fail to manifest as above-chance, since accuracies tend to concentrate and fluctuate around the chance level.

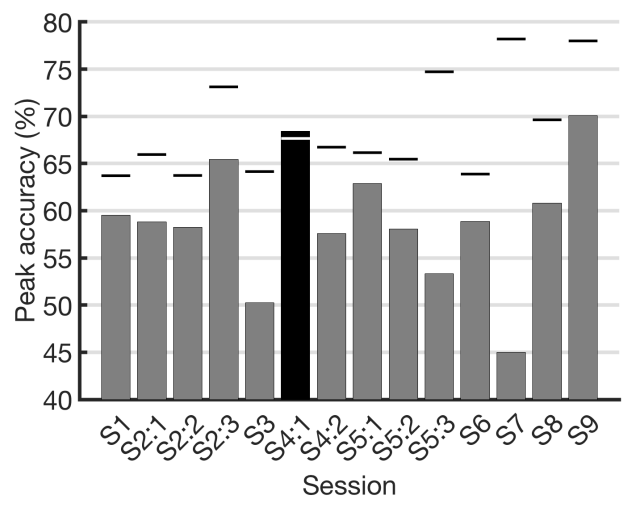

Fig. 3. Peak classification accuracy for each recording session. For patients with multiple recordings, the session is denoted as number after the colon. Black bars indicate sessions that reached a peak accuracy better than the 99\% chance level which is marked with a thin horizontal line for each run.
Hence, this criterion potentially generates false negatives for patients that exhibit physiological EEG correlates of motor activity. In [10], the accuracy in a motor imagery task yielded fewer positives compared to a language and a music task on the same population. In our own dataset, only 1 out of the expected 8 patients that have been identified as CMD by the MBT complied with the accuracy criterion (Fig. 3). Furthermore, accuracy values (irrespectively of significance) do not correlate with awareness, as approximated by the CRS-R (Fig. 2B).

We assumed that the maximum achieved discriminancy on spatio-spectral EEG correlates of motor attempt will be a better index of awareness. Our results seem to support this hypothesis, as the derived PSDP index correlates significantly with the CRS-R (Fig. 2A). A clear indicator for the detected EEG activity indeed coming from attempted movements is given by the prominent ERDs observed in the topographical maps of Fig. 4 for those subjects that also demonstrated high $r^{2}$ discriminancy. Albeit smaller and less focused than in a healthy population [16], the ERDs show an active involvement of the motor cortex in this group.

We postulate that the extracted PSDP index might be

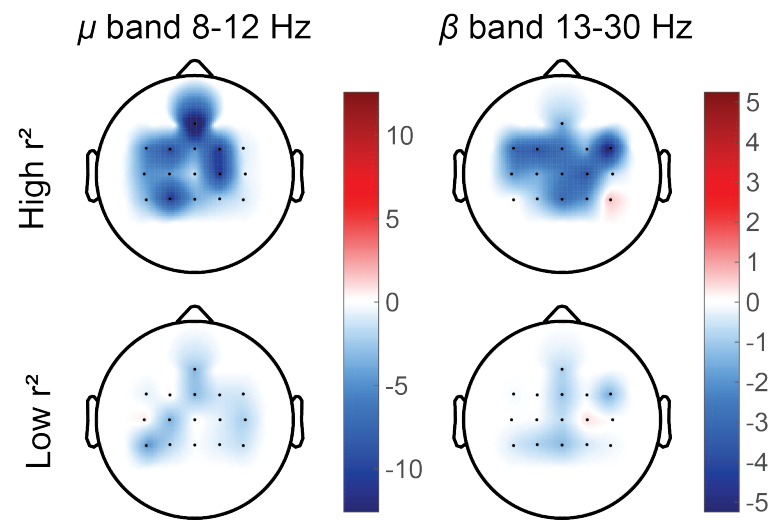

Fig. 4. Median event-related desynchronization (ERD) of patients with high or low $r^{2}$ index for the left hand motor task in the $\mu(8-12 \mathrm{~Hz})$ and $\beta(13-30 \mathrm{~Hz})$ band. The data for patients with a right hand motor task was mirrored at the sagittal plane. 
a better measure than the classification accuracy to study awareness in patients with DOC. The CRS-R and the PSDP strongly agree at the upper and lower bounds of the CRS-R scale, what validates the PSDP for undisputed cases. Interestingly, the CRS-R midrange values from ca. 7 to 16 seem to cluster into two distinct groups for the PSDP. It is exactly this range of CRS-R values where patients are thought to be often misclassified, usually towards underestimating their level of consciousness [1]. We posit that a threshold of PSDP around $r^{2} \approx 0.05$ separating the identified clusters could represent a boundary between conscious command-following behavior and unaware idling. In that aspect, it might serve both as additional evidence for the diagnosis of cases on the border between UWS and MCS, and as a tool to diagnose CMD.

It must be noted though, that the proposed PSDP criterion is not in perfect agreement with the behavioral MBT assessment. As can be seen in Fig. 2A, although this criterion seems to classify patient S3 as UWS (1 true negative, no false positives) and patients $\mathrm{S} 1, \mathrm{~S} 2, \mathrm{~S} 4, \mathrm{~S} 5$ and $\mathrm{S} 9$ as CMD (5 true positives) in accordance with the MBT scoring, the remaining three patients S6, S7 and S8 are seemingly misdiagnosed (3 false negatives). However, patient S6 suffered a second stroke during his stay in the rehabilitation unit prior to the EEG session, which might have overturned the original CMD diagnosis. Furthermore, these patients are known to suffer frequent lapses of attention and awareness [1], as also evident in the inter-session instability, so that additional EEG sessions probably would have changed this outcome.

Compared to the PSDP, a classification based on the accuracy criterion (see Fig. 3) indicates S4 as CMD and all others as UWS (1 true negative, 7 false negatives, 1 true positive, no false positives), showing that the PSDP outperforms accuracy also regarding the MBT.

The main limitations of this study are the currently very small sample size and the big UWS vs. CMD imbalance. Besides, the inconsistency of not collecting the CRS-R scores at the same day as the EEG session for every patient is now addressed and we assume that less time lag between EEG session and clinical scoring will further increase the found correlation between CRS-R and PSDP.

Our future work will seek to confirm these preliminary findings with more (in particular, UWS) patients and schedule multiple sessions to limit the chance of false negative outcomes. Additionally, we also plan to study the diagnostic utility of FES-induced cortical patterns and compare the FES feedback to similar published works that have relied only on auditory feedback.

\section{REFERENCES}

[1] J. T. Giacino, J. J. Fins, S. Laureys, and N. D. Schiff, "Disorders of consciousness after acquired brain injury: The state of the science," Nature Reviews Neurology, vol. 10, no. 2, pp. 99-114, 2014.

[2] S. Laureys, F. Pellas, P. Van Eeckhout, S. Ghorbel, C. Schnakers, F. Perrin, J. Berré, M. E. Faymonville, K. H. Pantke, F. Damas, M. Lamy, G. Moonen, and S. Goldman, "The locked-in syndrome: What is it like to be conscious but paralyzed and voiceless?" In Progress in Brain Research, vol. 150, Elsevier, 2005, pp. 495-511.
[3] R. T. Seel, M. Sherer, J. Whyte, D. I. Katz, J. T. Giacino, A. M. Rosenbaum, F. M. Hammond, K. Kalmar, T. L. B. Pape, R. Zafonte, R. C. Biester, D. Kaelin, J. Kean, and N. Zasler, "Assessment scales for disorders of consciousness: Evidence-based recommendations for clinical practice and research," Archives of Physical Medicine and Rehabilitation, vol. 91, no. 12, pp. 1795-1813, 2010.

[4] J. T. Giacino, K. Kalmar, and J. Whyte, "The JFK Coma Recovery Scale-Revised: Measurement characteristics and diagnostic utility," Archives of Physical Medicine and Rehabilitation, vol. 85, no. 12, pp. 2020-2029, 2004.

[5] J. T. Giacino, C. Schnakers, D. Rodriguez-Moreno, K. Kalmar, N. Schiff, and J. Hirsch, "Behavioral assessment in patients with disorders of consciousness: gold standard or fool's gold?" In Progress in Brain Research, vol. 177, Elsevier, 2009, pp. 33-48.

[6] S. Laureys and N. D. Schiff, "Coma and consciousness: Paradigms (re)framed by neuroimaging," Neurolmage, vol. 61, no. 2, pp. 478-491, 2012.

[7] N. D. Schiff and J. J. Fins, "Brain death and disorders of consciousness," Current Biology, vol. 26, no. 13, pp. 572576, 2016.

[8] C. L. Phillips, M. A. Bruno, P. Maquet, M. Boly, Q. Noirhomme, C. Schnakers, A. Vanhaudenhuyse, M. Bonjean, R. Hustinx, G. Moonen, A. Luxen, and S. Laureys, "“Relevance vector machine" consciousness classifier applied to cerebral metabolism of vegetative and locked-in patients," NeuroImage, vol. 56, no. 2, pp. 797-808, 2011.

[9] A. M. Owen, M. R. Coleman, M. Boly, M. H. Davis, S. Laureys, and J. D. Pickard, "Detecting Awareness in the Vegetative State," Science, vol. 313, no. 5792, p. 1402, 2006.

[10] B. L. Edlow, C. Chatelle, C. A. Spencer, C. J. Chu, Y. G. Bodien, K. L. O'Connor, R. E. Hirschberg, L. R. Hochberg, J. T. Giacino, E. S. Rosenthal, and O. Wu, "Early detection of consciousness in patients with acute severe traumatic brain injury," Brain, vol. 140, no. 9, pp. 2399-2414, 2017.

[11] J. D. Sitt, J. R. King, I. El Karoui, B. Rohaut, F. Faugeras, A. Gramfort, L. Cohen, M. Sigman, S. Dehaene, and L. Naccache, "Large scale screening of neural signatures of consciousness in patients in a vegetative or minimally conscious state," Brain, vol. 137, no. 8, pp. 2258-2270, 2014.

[12] S. L. Hauger, A.-K. Schanke, S. Andersson, C. Chatelle, C. Schnakers, and M. Løvstad, "The Clinical Diagnostic Utility of Electrophysiological Techniques in Assessment of Patients With Disorders of Consciousness Following Acquired Brain Injury," Journal of Head Trauma Rehabilitation, vol. 32, no. 3, pp. 185-196, 2017.

[13] S. Chennu, J. Annen, S. Wannez, A. Thibaut, C. Chatelle, H. Cassol, G. Martens, C. Schnakers, O. Gosseries, D. Menon, and S. Laureys, "Brain networks predict metabolism, diagnosis and prognosis at the bedside in disorders of consciousness," Brain, vol. 140, no. 8, pp. 2120-2132, 2017.

[14] C. Chatelle, S. Chennu, Q. Noirhomme, D. Cruse, A. M. Owen, and S. Laureys, "Brain-computer interfacing in disorders of consciousness," Brain Injury, vol. 26, no. 12, pp. 1510-1522, 2012.

[15] J. M. Pignat, E. Mauron, J. Jöhr, C. Gilart de Keranflec'h, D. Van De Ville, M. G. Preti, D. E. Meskaldji, V. Hömberg, S. Laureys, B. Draganski, R. Frackowiak, and K. Diserens, "Outcome Prediction of Consciousness Disorders in the Acute Stage Based on a Complementary Motor Behavioural Tool," PLoS ONE, vol. 11, no. 6, e0156882, 2016.

[16] G. Pfurtscheller and F. H. Lopes Da Silva, "Event-related EEG/MEG synchronization and desynchronization: Basic principles," Clinical Neurophysiology, vol. 110, no. 11, pp. 1842-1857, 1999. 